\title{
X-Ray-Induced Changes to Passivation Layers of Lithium-Ion Battery Electrodes
}

\author{
Benjamin T. Young $\mathbb{D}^{1},{ }^{1}$ David R. Heskett, ${ }^{2}$ Joseph C. Woicik, ${ }^{3}$ and Brett L. Lucht ${ }^{4}$ \\ ${ }^{1}$ Department of Physical Sciences, Rhode Island College, 600 Mount Pleasant Ave., Providence, RI 02908, USA \\ ${ }^{2}$ Department of Physics, University of Rhode Island, 2 Lippitt Rd., Kingston, RI 02881, USA \\ ${ }^{3}$ National Institute of Standards and Technology, 100 Bureau Dr., Gaithersburg, MD 20899, USA \\ ${ }^{4}$ Department of Chemistry, University of Rhode Island, 140 Flagg Rd., Kingston, RI 02881, USA \\ Correspondence should be addressed to Benjamin T. Young; byoung@ric.edu
}

Received 1 June 2018; Revised 4 September 2018; Accepted 16 September 2018; Published 1 November 2018

Academic Editor: Rizwan Hasan Khan

Copyright (C 2018 Benjamin T. Young et al. This is an open access article distributed under the Creative Commons Attribution License, which permits unrestricted use, distribution, and reproduction in any medium, provided the original work is properly cited.

\begin{abstract}
The surface sensitivity available to photoelectron spectroscopies (PESs) makes them popular techniques for characterization of chemical environments at shallower depths than other, more bulk-sensitive techniques and because they are generally thought to be nondestructive. Variable energy, synchrotron radiation (SR), permits access to information not available to common lab-based radiation sources, making high-energy PES studies extremely useful for understanding thin films and interfaces. High-SR photon flux has been useful for developing models of soft X-ray-induced effects, but hard X-ray SR-induced effects are less well studied and will be increasingly important as popularity and availability of SR for thin film analysis continues to grow. We report here on observed modification of the solid electrolyte interphase of a lithium-ion battery electrode during prolonged exposure to 4 keV SR. The effects can be summarized by desorption of oxygen-containing species from the sample surface and by reactions within the film. Also presented is an estimate of the layer thickness' time evolution during the prolonged SR exposure.
\end{abstract}

\section{Introduction}

Modifications of surfaces under spectroscopic investigations had been of interest, albeit mostly for technical manufacturing reasons [1], since before the observations of Redhead [2] and of Menzel and Gomer [3,4] in the 1960s. These studies reported desorption of ions of oxygen, hydrogen, carbon monoxide, and barium from adsorbed layers on metals under bombardment by low-energy electrons. The Menzel-Gomer-Redhead (MGR) model that developed in 1964 describes the mechanism responsible for their observations as a direct valence excitation that produces dissociation or desorption of the adsorbate, and the subsequent success of the MGR model helped electron-stimulated desorption (ESD) to become promising techniques for material investigations of many systems.

In the late 1970s and early 1980s, the Knotek-Feibelman (KF) model was developed to reinterpret ESD data that were not entirely consistent with the predictions of the MGR model [5-9]. The KF model identifies the primary process as core-level excitation, followed by Auger decay(s). This model is particularly suited to highly ionic systems and provides a natural extension to higher energy excitation, as well as to excitation by photons in photon-stimulated desorption (PSD), X-ray PSD (XPSD), and X-ray-induced electronstimulated desorption (XESD) studies.

The MGR and KF models as well as subsequent refinements to the theoretical understanding, including covalent systems [10], neutral fragments [11], lithography [12], defect-electron spreading [13], decomposition [14-17], and others have helped to maintain ESD/PSD popularity and utility for many years and have motivated the exploitation of the phenomena for various purposes. Urisu and coworkers demonstrated oxygen desorption from molybdenum oxide via synchrotron radiation (SR) as a viable means of surface cleaning [18], and a considerable focus on preferential 
photochemical reactions highlights the possibilities for deposition and doping in materials engineering applications [19-22].

Soft X-rays are common photon sources for these experiments as much of the theoretical interest involves excitation of core-levels with electron-binding energies in the sub-keV range. Chou and Wen note, however, that while many soft X-ray sources and their characteristic photon flux densities are generally assumed to cause negligible changes to the adsorbate in PSD studies, particular systems can exhibit variation in chemical-bonding structure, especially when high-intensity SR is used [23]. When hard X-ray sources are used, in addition to the MGR and KF model mechanisms, escaping photoelectrons contribute to a high-energy secondary electron background. As the energy of the exciting photons increases, in contrast to the KF model (where the energy of the generating Auger event is necessarily fixed) and the MGR model (where the cross section for direct ionization of valence electrons decreases), higher-energy electronstimulated effects will become more important. As Rosenberg and coworkers pointed out, the availability of high-energy SR motivates novel applications such as deep $\mathrm{X}$-ray lithography [24]. In that study, the authors demonstrated etching and deposition by irradiating substrates immersed in reactive solvents as well as fabrication of nanocrystalline particles. Core-level excitations from highenergy photons give enough energy to escaping photoelectrons to break chemical bonds, and the ability of the photons to modify the sample can scale with the energy.

Certainly, as the high energy and high flux of SR continue to find more applications in the fields of thin film systems and materials science at large, changes induced by the interaction of SR with the samples being studied will be important to consider. The deeper penetration of highenergy photons will make secondary, KF model-like, and other processes more important. Flege et al. have shown that $\mathrm{X}$-ray standing waves (XSW) using electrons and fluorescence photons as secondary signals with XPSD (XSW-PSD) is a viable tool to investigate desorption with the ability to identify the underlying direct or indirect processes in a sitespecific manner for several systems (see [25] and references therein) with photon energies in the HAXPES range. In the present work, we report on observations of structural and chemical changes to a complex passivation layer on a silicon nanoparticle substrate using $4 \mathrm{keV}$ SR.

The solid electrolyte interphase (SEI) is a passivation layer that grows on electrodes in lithium-ion batteries (LIBs) during normal charge/discharge cycling. Development of chemistries to promote a superior SEI is one part of the ongoing effort to improve secondary battery performance [26-28]. Many methods being employed to improve performance may be better understood by hard X-ray photoelectron spectroscopy (HAXPES) investigation or by the more common, lower energy version of this technique, XPS, which differs from HAXPES [29-33]. The SR-based HAXPES technique is able to provide additional information due to the higher-energy photons available from synchrotron sources [34-39]. Most lab-based XPS experiments provide photons with one or two distinct energies $(1487 \mathrm{eV} \mathrm{Al}-\mathrm{K} \alpha$ or $1254 \mathrm{eV} \mathrm{Mg-K} \alpha$ ), whereas SR gives access to photons with energy up to $10 \mathrm{keV}$. The greater energy given to escaping photoelectrons in HAXPES experiments provides information from greater mean depth in the SEI than what is obtained from XPS (roughly double, in this investigation), and the tunability of SR enables characterization of the SEI at multiple depths in HAXPES experiments. Included in the supplemental information for this report is a comparison of the mean free path for core electrons in lab-based XPS instruments with HAXPES experiments.

The HAXPES technique can be extremely useful to the LIB effort, but care must be taken to understand the effects of SR irradiation, especially as they concern modifications to the SEI. Edstrom et al. note that attention was paid to these concerns by monitoring the $\mathrm{C} 1 \mathrm{~s}$ and $\mathrm{F} 1 \mathrm{~s}$ spectra during data collection and decreasing the radiation intensity [35]. However, to our knowledge, neither the nature nor the extents of the X-ray-induced changes we report here have been systematically studied.

\section{Materials and Methods}

For the present study, we analyzed a binder-free silicon nanoparticle electrode after 20 charge/discharge cycles in a coin-cell Li-ion battery built with $1.2 \mathrm{M} \mathrm{LiPF}_{6}$ electrolyte salt in fluoroethylene carbonate (FEC) as solvent (half-cell). Preparation, charge/discharge cycling, and disassembly of the battery from which the sample analyzed in this study was harvested has been previously published in our study regarding the formation of the SEI for this system [37]. The harvested electrode was dried overnight in the vacuum antechamber of the Ar-filled glovebox in which the battery was disassembled, installed in a tightly sealed vessel under Ar at atmospheric pressure, and transported to the X-24A endstation of the NIST beamline at the National Synchrotron Light Source (NSLS-I), Brookhaven National Laboratory (Upton, NY), for analysis. At the beamline, the vessel was opened in a glovebag attached to the fast-turnaround load lock port of the endstation as a positive pressure of $\mathrm{N}_{2}$ was flown outward through the bag. We recorded spectra for the $\mathrm{C} 1 \mathrm{~s}, \mathrm{P} 1 \mathrm{~s}, \mathrm{Si} 1 \mathrm{~s}$, and $\mathrm{O} 1 \mathrm{~s}$ cores in that order and spending equal time scanning each region, so one complete collection period took 25 minutes. This time is roughly what it takes to collect all cores for a given sample at a given photon energy in one of our HAXPES studies. To elucidate the changes SR induced, we repeated 25-minute collection periods back to back for a total SR exposure of more than three hours.

Estimates for the SEI layer's relative thickness during SR irradiation are performed using the Si 1s core signal, similar to our previous work [39], but since there is no unchanging species that is localized to the substrate and obviously apparent in this system, as the metal oxide signal was in that study, we have reported thickness estimates relative to the initial measurements of two peaks $\left(\mathrm{Li}_{\mathrm{x}} \mathrm{SiO}_{\mathrm{y}}\right.$ and $\left.\mathrm{Li}_{\mathrm{x}} \mathrm{Si}\right)$ in the Si 1s spectrum representing the interface between the SEI and the substrate silicon nanoparticles. 

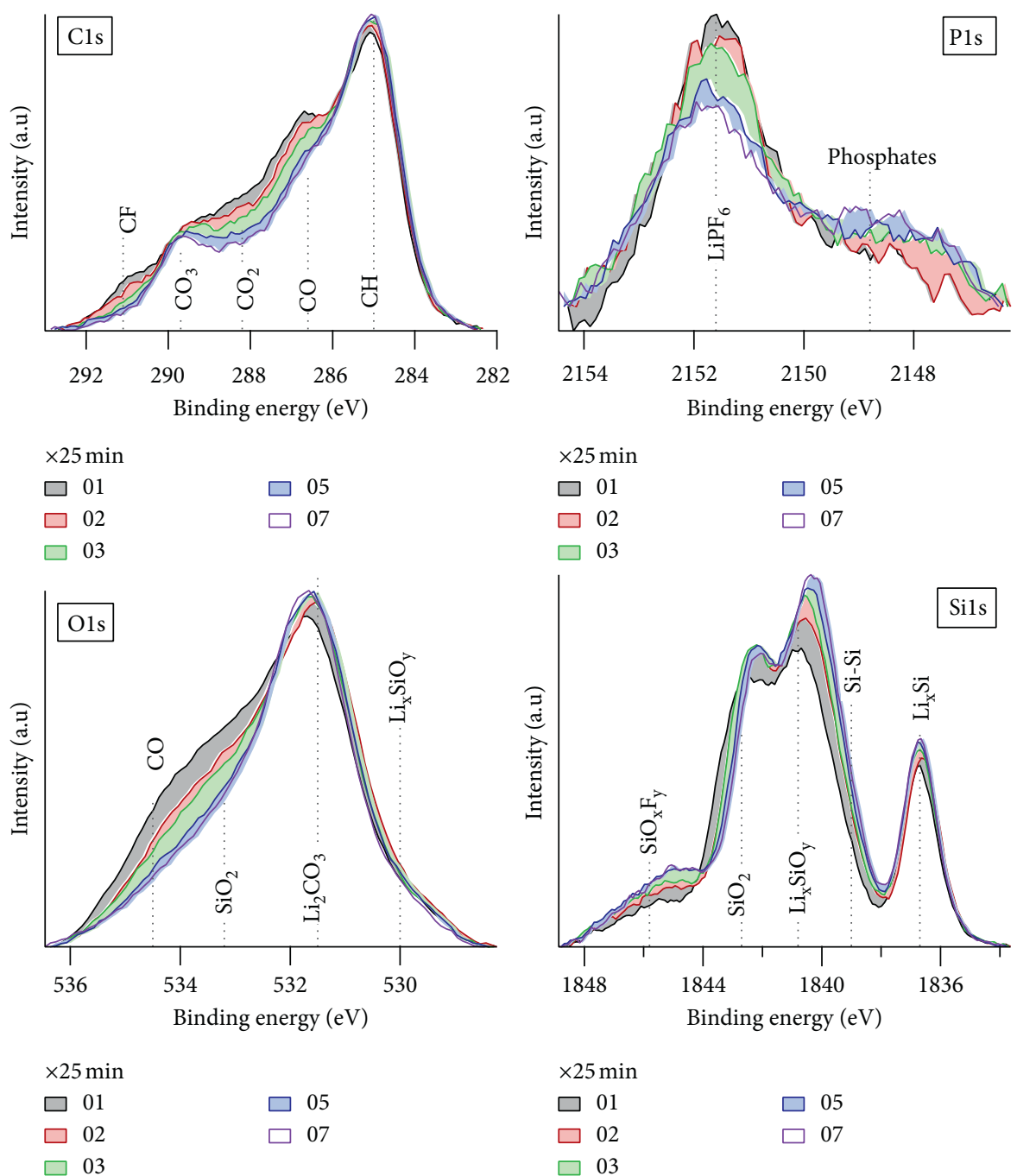

FIgURE 1: Individual 1s electron spectra for (CW from top left) C, P, Si, and O for BF-Si nanoparticle electrode after 20 charge/discharge cycles in FEC solvent, displayed in increments of $25 \mathrm{~min}$. of exposure to $4 \mathrm{keV}$ photon flux ( $200 \mathrm{~J}$ of energy). Noise in the P $1 \mathrm{~s}$ spectrum is due to the small concentration of phosphorus in the SEI and the desire to minimize data collection time.

The thickness approximation is described by the following equation:

$$
\frac{C_{i}}{C_{f}}=\frac{\int_{d}^{\infty} e^{-(x / \lambda \sin \theta)} d x}{\int_{0}^{\infty} e^{-(x / \lambda \sin \theta)} d x} \approx e^{-(d / \lambda)},
$$

where the ratio of the concentration $C_{i}$ in a given sample to the concentration $C_{f}$ in the fresh sample equates to an exponential which is readily solved for $d$, the thickness of the sample. The inelastic mean free path of polyethylene was used for $\lambda[40]$. The angle between the analyzer line of sight and the surface normal to the sample defines $\theta$, which was fixed at $3^{\circ}$. Concentrations were calculated according to the following equation:

$$
C_{i}=\frac{A_{i} /\left(T(K E)_{i} \sigma(h v)_{i} \lambda(K E)_{i}\right)}{\left(\sum_{j} A_{j} /\left(T(K E)_{j} \sigma(h v)_{j} \lambda(K E)_{j}\right)\right)}
$$

where $A$ corresponds to the spectrum area after Shirley background subtraction and normalization to the background level, $T$ is the relative transmission function of the X-24A analyzer [41], $\sigma$ is the Scofield photoionization cross section [42], $\lambda$ is defined as above, and the summation index $j$ runs over all distinct elements analyzed. The Si 1s concentration was separated for this analysis and restricted to include only the areas of the $\mathrm{Li}_{x} \mathrm{SiO}_{y}$ and $\mathrm{Li}_{\mathrm{x}} \mathrm{Si}$ peak contributions, as obtained through fits to the experimental data using Voigt shapes.

\section{Results and Discussion}

While there were no observable changes to the spectra between individual spot tests at different locations on the sample and the first displayed period of data collection, we observe changes to all collected spectra between the first and second periods of data collection. Selected spectra are presented in Figure 1 as plots of intensity versus electronbinding energy in $\mathrm{eV}$. The spectra are arrayed in four panes, each representing one of the four cores, and each pane 
displays the progression of the spectrum relative to the time of beam exposure. Several traces are displayed for time intervals between one and seven multiples of 25 minutes, representing the beginning of each four-core period of data collection. We saw no obvious further changes to the spectra for beam exposure times longer than 175 minutes.

The most striking features in Figure 1 are observed in the $\mathrm{C}$ 1s (upper left) and O 1s (lower left) spectra. In the carbon spectrum, there is a very clear reduction in the signals we have assigned to species with C-O $(286.5 \mathrm{eV})$, $\mathrm{CO}_{3}(289.7 \mathrm{eV})$, and C-F $(291 \mathrm{eV})$ bonds. In the oxygen spectra, the major feature is the reduced intensity of the shoulder on the high-binding energy-side of the broad peak. We assign this shoulder to the $\mathrm{C}-\mathrm{O}$ bond (binding energy $534.5 \mathrm{eV}$ ), consistent with the concomitant change in the carbon spectra. These changes can be explained by desorption of oxygen-containing species from the surface layer. That the $\mathrm{Li}_{2} \mathrm{CO}_{3}$ intensity is not obviously changed in the carbon and oxygen spectra supports the suggestion from our previous work that this species is more prevalent deeper in the SEI [37] and/or is less sensitive to beam exposure. The reduction of the C-F signal intensity at $291 \mathrm{eV}$ is likely due to carbonization of sequestered FEC (or its decomposition products [43]) similar to what has been reported for observations on the polymer polyvinylidene fluoride, a very popular binder material for $\mathrm{Li}$-ion electrode fabrication $[33,36]$, upon exposure to soft X-rays $[15,16]$. In Figure 2, the integrated intensity of each spectrum is plotted against exposure time, and the curves are normalized to the initial observed intensity for each element. The carbon and oxygen traces both decrease in a roughly linear, nearly identical fashion in time consistent with photonstimulated desorption proportional to the energy delivered to the sample by a photon beam of constant energy and nearly constant flux.

We estimate the energy incident on the sample during each 25-minute data collection period to be roughly $200 \mathrm{~mJ}$ (at a flux of approximately $2.1 \times 10^{12}$ photon s$^{-1}$ ) as an upper limit based on published photon flux ranges delivered to the beamline from the storage ring, the beamline optics, as well as the geometry of the sample and its orientation relative to the photon beam [44-46].

By contrast, the phosphorus trace in Figure 2 indicates very little change in total intensity versus time. The phosphorus trend is less clear than the other three traces in Figure 2 owing to the relatively lower signal/noise for $\mathrm{P} 1 \mathrm{~s}$ spectra of Figure 1. This is to be expected as less than 1\% of the total signal observed comes from phosphoruscontaining species, and the collection time was kept constant across all four cores to minimize the collection period. Inspecting the $\mathrm{P} 1 \mathrm{~s}$ progression in the top right pane of Figure 1, however, reveals the relatively constant total (integrated) phosphorus signal is a result of intensity redistribution indicating recombination. The $\mathrm{P}$ 1s spectra, show a clear reduction in intensity of the higher-binding energy peak attributed to unmodified electrolyte salt $\mathrm{LiPF}_{6}$ $(2151.5 \mathrm{eV})$ trapped in the SEI, and an increase in the intensity of the broad peak at lower binding energy

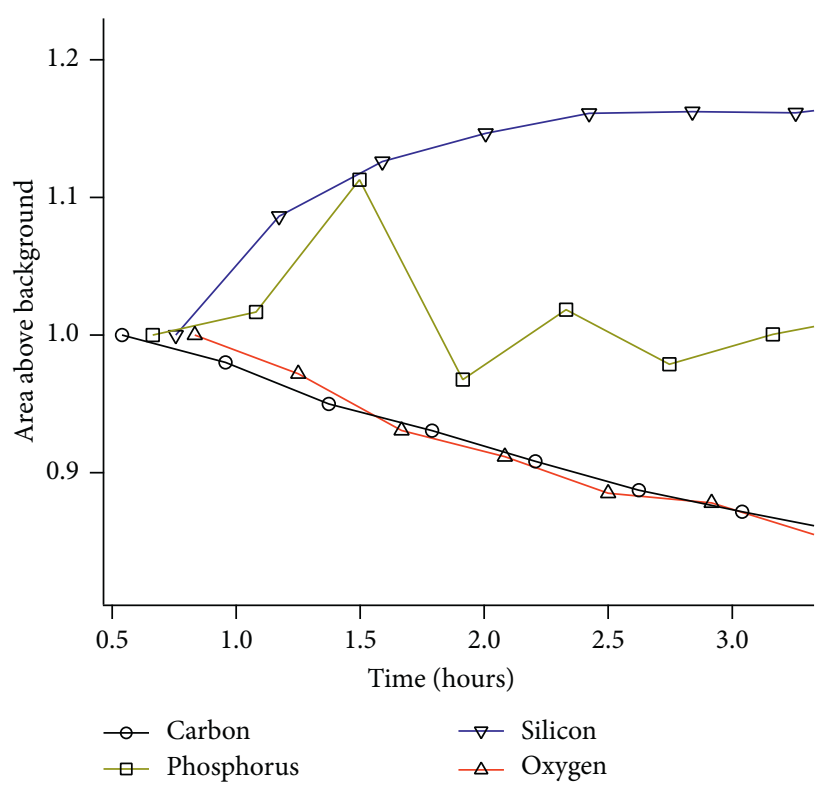

FIgURE 2: Total signal intensity vs. beam exposure time for the $1 \mathrm{~s}$ electrons of $\mathrm{Si}, \mathrm{P}, \mathrm{O}$, and $\mathrm{C}$ normalized to the initial intensity from BF-Si nanoparticle battery electrode after 20 charge/discharge cycles in FEC solvent. Uncertainty in these measurements, propagated from the fit areas, is less than $10 \%$ for all measurements. Energy incident on the sample from the $4 \mathrm{keV}$ photon beam is estimated to be $1.5 \mathrm{~J}$ after 3 hours.

assigned to fluorinated lithium phosphates. This result is consistent with photon-stimulated decomposition of the $\mathrm{LiPF}_{6}$ salt, as phosphates are an expected product of its decomposition [47].

The total intensity for silicon species in Figure 2 is nonlinear in time, increasing dramatically for the first few 25-minute measurement periods and less so for further irradiation, and finally approaching an apparent asymptote for exposure longer than about two hours, which we estimate to be equivalent to $1 \mathrm{~J}$ of absorbed energy from the $4 \mathrm{keV}$ photon beam. This result is mirrored in the Si 1s spectra displayed in Figure 1, where all peaks appear to gain some intensity in time with nothing obviously decreasing. Desorption from the SEI indicated in the carbon and oxygen spectra may impact the layer thickness to such an extent that more of the substrate silicon is visible to the technique, which is consistent with the apparent intensity increases of all identified silicon-containing species. Estimation of the relative thickness decrease of the SEI layer is reported in Figure 3 as calculated from Voigt fits to the peaks in the Si 1 s spectra we have identified as $\mathrm{Li}_{\mathrm{x}} \mathrm{Si}$ and $\mathrm{Li}_{\mathrm{x}} \mathrm{SiO}_{\mathrm{y}}$. The measurements from the two peaks agree well within the uncertainty of the measurements. We estimate that the SEI thickness is decreased by about $1 \mathrm{~nm}$ after three hours of SR irradiation $(\sim 1.5 \mathrm{~J})$ but at that point the thickness does not appear to be markedly changing. We have recently estimated the SEI thickness developed on different systems [39] and found the total SEI thickness to be on the order of tens of nanometers. 


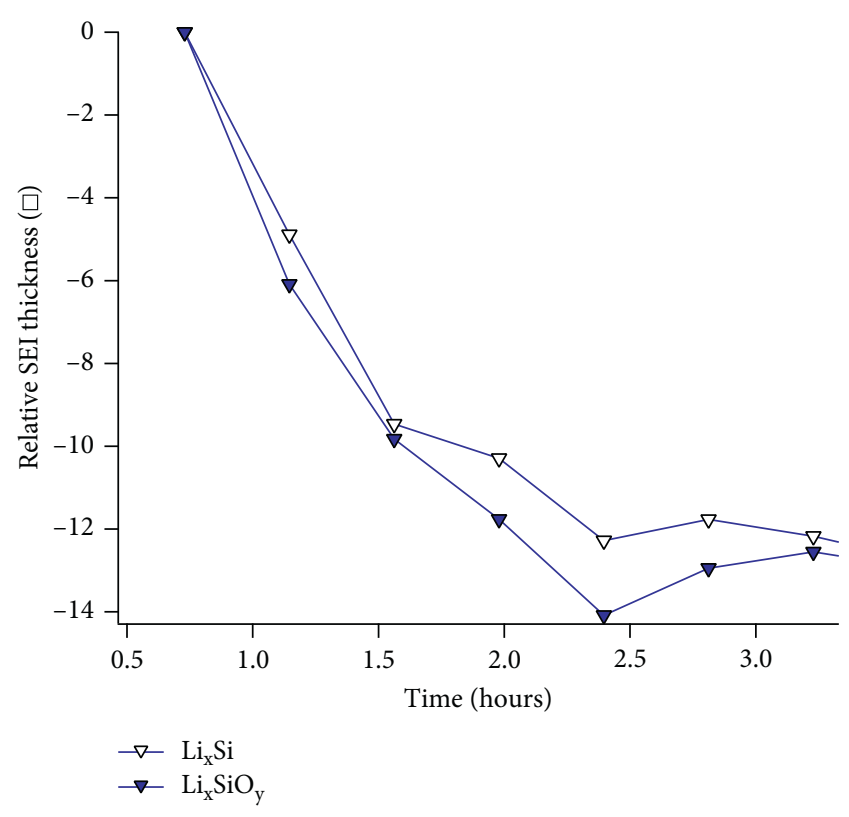

FIGURE 3: Thickness estimates for the SEI calculated relative to the initial measurement of the $\mathrm{Si} 1$ s core excited with $4 \mathrm{keV}$ photons for the peak we identified as $\mathrm{Li}_{\mathrm{x}} \mathrm{Si}$ (open markers) and $\mathrm{Li}_{\mathrm{x}} \mathrm{SiO}_{\mathrm{y}}$ (solid markers). Uncertainty in these estimates, propagated from the fit areas, is less than $10 \%$ for all measurements. Energy incident on the sample from the $4 \mathrm{keV}$ photon beam is estimated to be $1.5 \mathrm{~J}$ after 3 hours.

\section{Conclusions}

We have analyzed the evolution of a complex, passivating SEI layer grown on the BF-Si electrode of a LIB after 20 charge/discharge cycles to document the changes that are observed due only to SR irradiation during ex situ analysis. We have observed modification of the SEI consistent with desorption of oxygen- and carboncontaining species as well as conversion between phosphorus-containing species. We are able to estimate how much thickness of the layer is lost to desorption and find it to be slightly more than $1 \mathrm{~nm}$ after more than three hours of SR irradiation, which we estimate to be equivalent to $1.5 \mathrm{~J}$ of energy from the $4 \mathrm{keV}$ photon beam. Relative to the estimated thickness of SEIs from similar systems, this loss accounts for less than $5 \%$ of the total layer thickness for exposure to SR well in excess of our usual data collection time.

\section{Data Availability}

The raw HAXPES spectral data files used to support the findings of this study are available from the corresponding author upon request.

\section{Conflicts of Interest}

The authors declare that there are no conflicts of interest regarding the publication of this paper.

\section{Acknowledgments}

BTY would like to thank Drs. Jamie Towle-Weicksel and Laura Cooley at Rhode Island College for illuminating discussions. Use of the National Synchrotron Light Source, Brookhaven National Laboratory, was supported by the U.S. Department of Energy, Office of Science, Office of Basic Energy Sciences (Contract No. DE-AC02-98CH10886). We gratefully acknowledge funding from the Department of Energy, Office of Basic Energy Sciences (EPSCoR Implementation award DE-SC0007074). Additional support was provided by the National Institute of Standards and Technology, and to BTY through a Rhode Island College Faculty Research Committee Grant.

\section{Supplementary Materials}

In addition to a graphic highlighting the general mean free path length increase for synchrotron-based HAXPES over lab-based XPS photoelectrons, the supplemental material is included to support atomic concentrations and relative SEI thickness calculations used in the manuscript. There is a page of constants and fit parameters referenced but not explicitly reported in the main text, as well as specific mean free path comparisons for several core electrons, and a page containing data from fits to spectra in Figure 1 that were used to create Figure 3 in the manuscript. (Supplementary Materials)

\section{References}

[1] P. A. Redhead, "The first 50 years of electron stimulated desorption (1918-1968)," Vacuum, vol. 48, no. 6, pp. 585-596, 1997.

[2] P. A. Redhead, "Interaction of slow electrons with chemisorbed oxygen," Canadian Journal of Physics, vol. 42, no. 5, pp. 886-905, 1964.

[3] D. Menzel and R. Gomer, "Desorption from metal surfaces by low-energy electrons," Journal of Chemical Physics, vol. 41, no. 11, pp. 3311-3328, 1964.

[4] D. Menzel and R. Gomer, "Electron-impact desorption of carbon monoxide from tungsten," Journal of Chemical Physics, vol. 41, no. 11, pp. 3329-3351, 1964.

[5] P. J. Feibelman and M. L. Knotek, "Reinterpretation of electron-stimulated desorption data from chemisorption systems," Physical Review B, vol. 18, no. 12, pp. 6531-6539, 1978.

[6] M. L. Knotek, V. O. Jones, and V. Rehn, "Photon-stimulated desorption of ions," Physical Review Letters, vol. 43, pp. 300-303, 1979.

[7] M. L. Knotek, V. O. Jones, and V. Rehn, "Photon-stimulated desorption by core level excitation: density of states and extended fine structures," Surface Science, vol. 102, no. 2-3, pp. 566-577, 1981.

[8] P. J. Feibelman, "Reneutralization bottleneck in auger initiated desorption," Surface Science, vol. 102, no. 2-3, pp. L51L53, 1981.

[9] R. Jaeger, J. Stöhr, J. Feldhaus, S. Brennan, and D. Menzel, "Photon-stimulated desorption following deep core-level excitation: O on Mo(100)," Physical Review B, vol. 23, no. 5, pp. 2102-2110, 1981. 
[10] D. E. Ramaker, "Covalent interaction effects in electron/ photon-stimulated desorption," Journal of Vacuum Science and Technology A: Vacuum, Surfaces, and Films, vol. 1, no. 2, pp. 1137-1144, 1982.

[11] S. P. Frigo, P. Feulner, B. Kassühlke, C. Keller, and D. Menzel, "Observation of neutral atomic fragments for specific 1s core excitations of an adsorbed molecule," Physical Review Letters, vol. 80, no. 13, pp. 2813-2816, 1998.

[12] T. Ito, M. Gotoh, K. Sudoh, and H. Iwasaki, "Quantum yield of electron-beam induced decompostion of $\mathrm{Sio}_{2}$ overlay on $\mathrm{Si}$ in nanolithography using a scanning tunneling microscope," Japanese Journal of Applied Physics, vol. 40, no. 10, pp. 6055-6058, 2001.

[13] Z. Zhang, K. Cao, and J. T. Yates Jr., "Defect-electron spreading on the tio2(110) semiconductor surface by water adsorption," Journal of Physical Chemistry Letters, vol. 4, no. 4, pp. 674-679, 2013.

[14] H. S. Tao, U. Diebold, V. Chakarian et al., "Radiation-induced decomposition of PF3 on $\mathrm{Ru}(0001)$," Journal of Vacuum Science and Technology A: Vacuum, Surfaces, and Films, vol. 13, no. 5, pp. 2553-2557, 1995.

[15] J. A. M. Kuvshinov, S. S. Chebotaryov, L. A. Pesin et al., "Kinetic of poly(vinylidene fluoride) defluorination under X-ray irradiation," Journal of Surface Investigation. X-ray, Synchrotron and Neutron Techniques, vol. 4, no. 1, pp. 122127, 2010.

[16] L. A. Pesin, S. S. Chebotaryov, A. M. Kuvshinov et al., "Electron emission features of the derivatives of radiation carbonization of poly(vinylidene fluoride)," Journal of Surface Investigation. X-ray, Synchrotron and Neutron Techniques, vol. 4, no. 2, pp. 214-220, 2010.

[17] I. V. Voinkova, N. N. Ginchitskii, I. V. Gribov et al., "A model of radiation-induced degradation of the poly(vinylidene fluoride) surface during XPS measurements," Polymer Degradation and Stability, vol. 89, no. 3, pp. 471-477, 2005.

[18] M. Nagase, Y. Utsumi, H. Akawaza, and T. Urisu, "Oxygen desorption from molybdenum oxide by synchrotron radiation and its surface-cleaning applications," Applied Physics Letters, vol. 62, no. 3, pp. 234-236, 1992.

[19] D. R. Strongin and J. F. Moore, "Synchrotron radiation assisted deposition of aluminum oxide from condensed layers of trimethylaluminum and water at $78 \mathrm{~K}$," Applied Physics Letters, vol. 61, no. 6, pp. 729-731, 1992.

[20] R. A. Rosenberg, S. P. Frigo, S. Lee, and A. Dowben, "Selective area, synchrotron radiation induced, delta doping of silicon," Journal of Applied Physics, vol. 71, no. 10, pp. 4795-4798, 1992.

[21] R. A. Rosenberg, F. K. Perkins, D. C. Mancini et al., "Selective area deposition of boron on $\mathrm{Si}(111)$ induced by synchrotron radiation," Applied Physics Letters, vol. 58, no. 6, pp. 607-609, 1991.

[22] S. L. Kollmannsberger, C. A. Walenta, A. Winnerl et al., "Doping-dependent adsorption and photon-stimulated desorption of CO on Gan(0001)," Journal of Physical Chemistry C, vol. 121, no. 15, pp. 8473-8479, 2017.

[23] L. C. Chou and C. R. Wen, "Continuous-time core-level photon-stimulated desorption spectroscopy for monitoring soft X-ray induced reactions of molecules adsorbed on a single-crystal surface," Physical Review B, vol. 73, no. 19, article 195407, 2006.

[24] R. A. Rosenberg, Q. Ma, B. Lai, and D. C. Mancini, "Surface photochemistry induced by X-ray irradiation," Journal of Vacuum Science and Technology B: Microelectronics and Nanometer Structures, vol. 16, no. 6, pp. 3535-3538, 1998.
[25] J. I. Flege, T. Schmidt, A. Hille, J. Falta, and G. Materlik, "Photon-stimulated desorption and x-ray standing waves," Synchrotron Radiation News, vol. 70, no. 3, pp. 43-47, 2004.

[26] J. B. Goodenough and K. S. Park, "The Li-ion rechargeable battery: a perspective," Journal of the American Chemical Society, vol. 135, no. 4, pp. 1167-1176, 2013.

[27] K. Xu, "Electrolytes and interphases in Li-ion batteries and beyond," Chemical Reviews, vol. 114, pp. 11503-11618, 2014.

[28] N. Nitta, F. Wu, J. T. Lee, and G. Yushin, "Li-ion battery materials: present and future," Materials Today, vol. 18, no. 5, pp. 252-264, 2015.

[29] H. Nakai, T. Kubota, A. Kita, and A. Kawashima, "Investigation of the solid electrolyte interphase formed by fluoroethylene carbonate on Si electrodes," Journal of the Electrochemical Society, vol. 158, no. 7, pp. A798-A801, 2015.

[30] L. Bodenes, R. Dedryvère, H. Martinez, F. Fischer, C. Tessier, and J. P. Pèrès, "Lithium-ion batteries working at $85^{\circ} \mathrm{C}$ : aging phenomena and electrode/electrolyte interfaces studied by XPS," Journal of the Electrochemical Society, vol. 159, no. 10, pp. A1739-A1746, 2012.

[31] N. S. Choi, K. H. Yew, K. Y. Lee, M. Sung, H. Kim, and S. S. Kim, "Effect of fluoroethylene carbonate additive on interfacial properties of silicon thin-film electrode," Journal of Power Sources, vol. 161, no. 2, pp. 1254-1259, 2006.

[32] S. Dalavi, P. Guduru, and B. L. Lucht, "Performance enhancing electrolyte additives for lithium ion batteries with silicon anodes," Journal of the Electrochemical Society, vol. 159, no. 5, pp. A642-A646, 2012.

[33] S. H. Kang, D. P. Abraham, A. Xiao, and B. L. Lucht, "Investigating the solid electrolyte interphase using binder-free graphite electrodes," Journal of Power Sources, vol. 175, no. 1, pp. 526-532, 2008.

[34] C. S. Fadley, "X-ray photoelectron spectroscopy: progress and perspectives," Journal of Electron Spectroscopy and Related Phenomena, vol. 178-179, pp. 2-32, 2010.

[35] S. Malmgren, K. Ciosek, M. Hahlin et al., "Comparing anode and cathode electrode/electrolyte interface composition and morphology using soft and hard X-ray photoelectron spectroscopy," Electrochimica Acta, vol. 97, pp. 23-32, 2013.

[36] M. Nie, J. Demeaux, B. T. Young et al., "Effect of vinylene carbonate and fluoroethylene carbonate on SEI formation on graphitic anodes in Li-ion batteries," Journal of the Electrochemical Society, vol. 162, no. 13, pp. A7008-A7014, 2015.

[37] B. T. Young, D. R. Heskett, C. C. Nguyen, M. Nie, J. C. Woicik, and B. L. Lucht, "Hard X-ray photoelectron spectroscopy (HAXPES) investigation of the silicon solid electrolyte interphase (SEI) in lithium-ion batteries," ACS Applied Materials and Interfaces, vol. 7, pp. 20004-20011, 2015.

[38] D. M. Seo, C. C. Nguyen, B. T. Young, D. R. Heskett, J. C. Woicik, and B. L. Lucht, "Characterizing solid electrolyte interphase on Sn anode in lithium ion battery," Journal of the Electrochemical Society, vol. 162, no. 13, pp. A7091-A7095, 2015.

[39] Y. Dong, B. T. Young, Y. Zhang et al., "Effect of lithium borate additives on cathode film formation in $\operatorname{Lini}_{0.5} \mathrm{Mn}_{1.5} \mathrm{O}_{4} / \mathrm{Li}$ cells," ACS Applied Materials and Interfaces, vol. 9, no. 24, pp. 20467-20475, 2017.

[40] L. R. Painter, E. T. Arakawa, M. W. Williams, and J. C. Ashley, "Optical properties of polyethylene: measurement and applications," Radiation Research, vol. 83, no. 1, pp. 1-18, 1980.

[41] C. Weiland, R. Browning, B. A. Karlin, D. A. Fischer, and J. C. Woicik, "Note: alignment/focus dependent core-line sensitivity for quantitative chemical analysis in hard X-ray 
photoelectron spectroscopy using a hemispherical electron analyzer," Review of Scientific Instruments, vol. 84, article 036106, 2013.

[42] J. H. Scofield, Theoretical Photoionization Cross Sections from 1 to $1500 \mathrm{Kev}$; UCRL-51326, 4545040, Lawrence Livermore Laboratory, Livermore, CA, USA, 1973.

[43] G. M. Veith, M. Doucet, R. L. Sacci, B. Vacaliuc, J. K. Baldwin, and J. F. Browning, "Determination of the solid electrolyte interphase structure grown on a silicon electrode using a fluoroethylene carbonate additive," Scientific Reports, vol. 7, no. 1, p. 6236, 2017.

[44] P. L. Cowan, S. Brennan, R. D. Deslattes, A. Henins, T. Jach, and E. G. Kessler, "A high energy resolution X-ray spectroscopy synchrotron radiation beamline for the energy range 800-5000 ev," Nuclear Instruments and Methods in Physics Research Section A: Accelerators, Spectrometers, Detectors and Associated Equipment, vol. 246, no. 1-3, pp. 154-158, 1986.

[45] P. L. Cowan, S. Brennan, T. Jach, D. W. Lindle, and B. A. Karlin, "Performance of a high-energy-resolution, tender X-ray synchrotron radiation beamline (invited)," Review of Scientific Instruments, vol. 60, no. 7, pp. 1603-1607, 1989.

[46] C. Weiland, A. K. Rumaiz, P. Lysaght, B. Karlin, J. C. Woicik, and D. Fischer, "NIST high throughput variable kinetic energy hard X-ray photoelectron spectroscopy facility," Journal of Electron Spectroscopy and Related Phenomena, vol. 190, pp. 193-200, 2013.

[47] C. L. Campion, W. Li, and B. L. Lucht, "Thermal decomposition of $\mathrm{LiPF}_{6}$ - based electrolytes for lithium-ion batteries," Journal of The Electrochemical Society, vol. 152, no. 12, pp. A2327-A2334, 2005. 

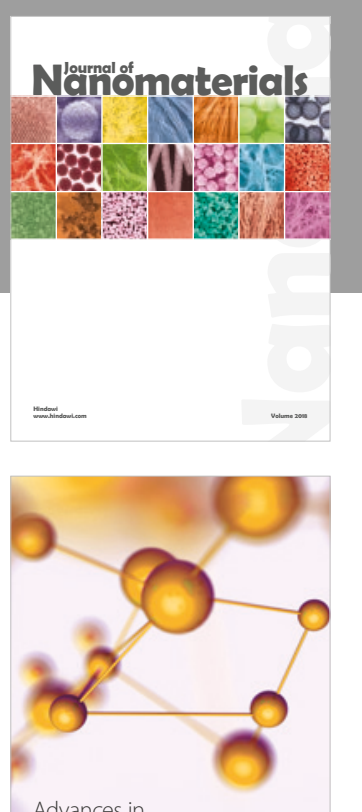

Physical Chemistry
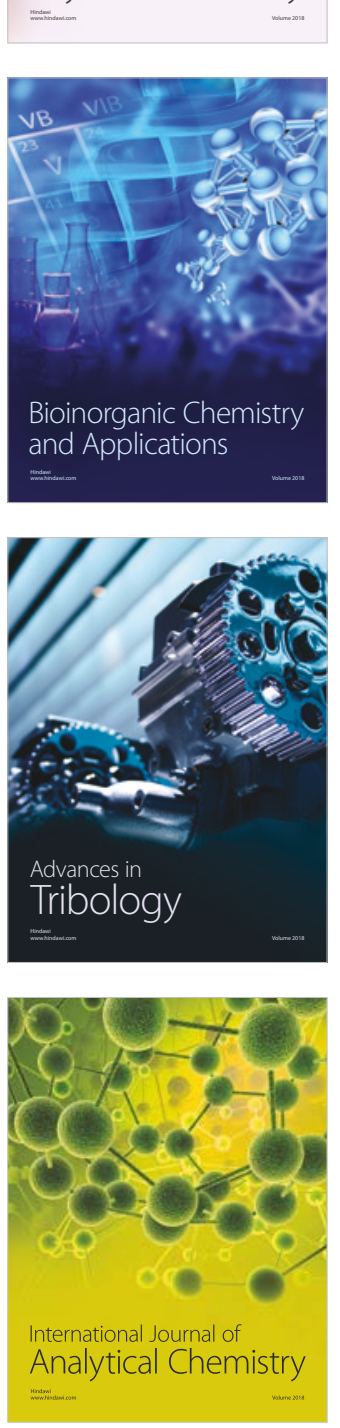

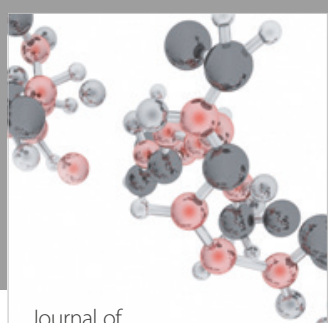

Analytical Methods

in Chemistry

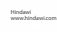

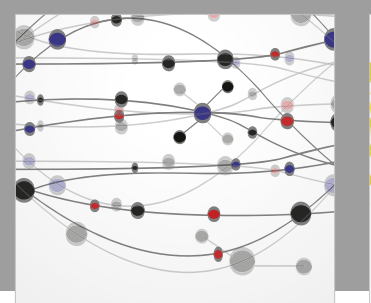

The Scientific World Journal

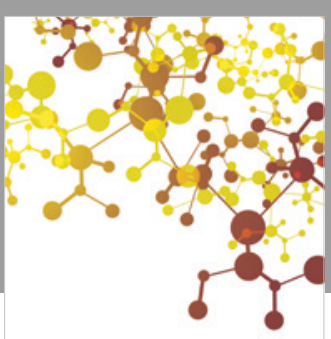

Journal of

Applied Chemistry
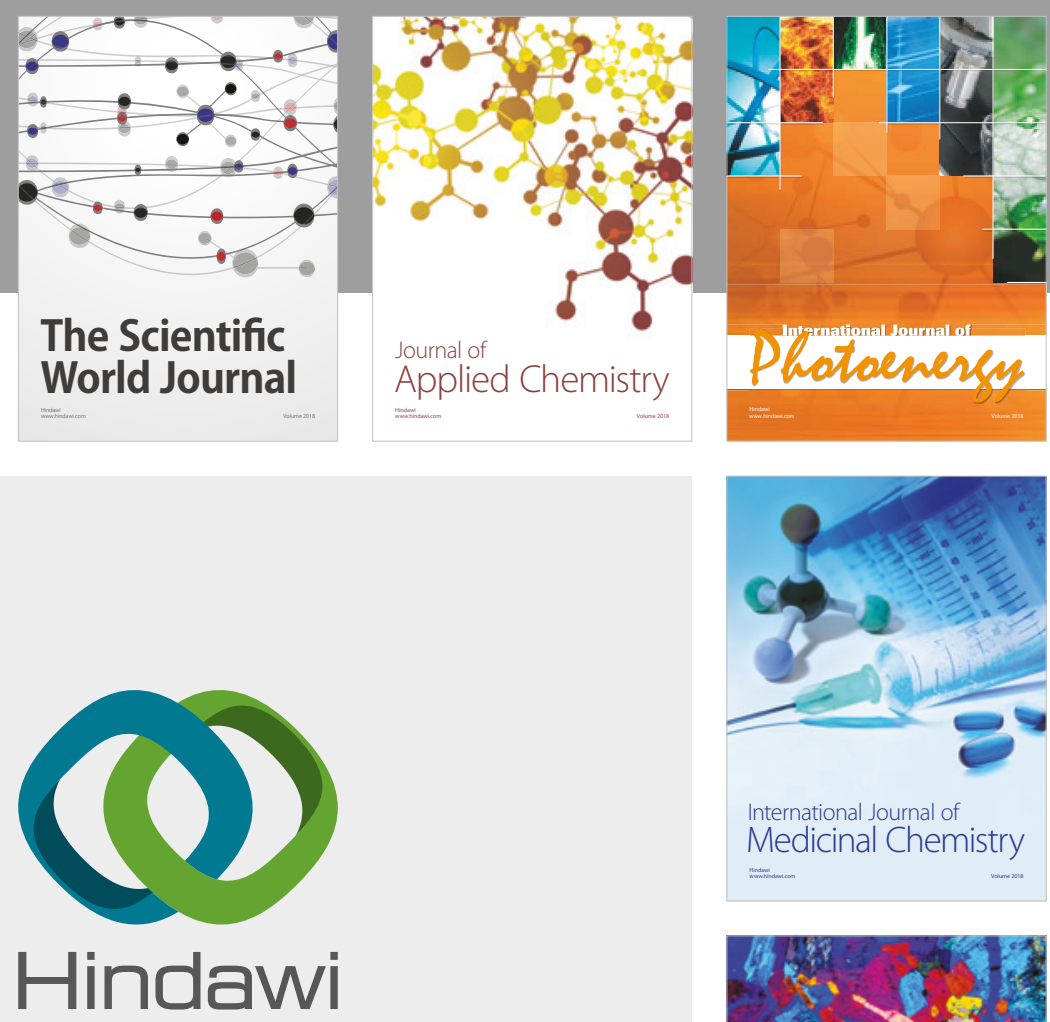

Submit your manuscripts at

www.hindawi.com
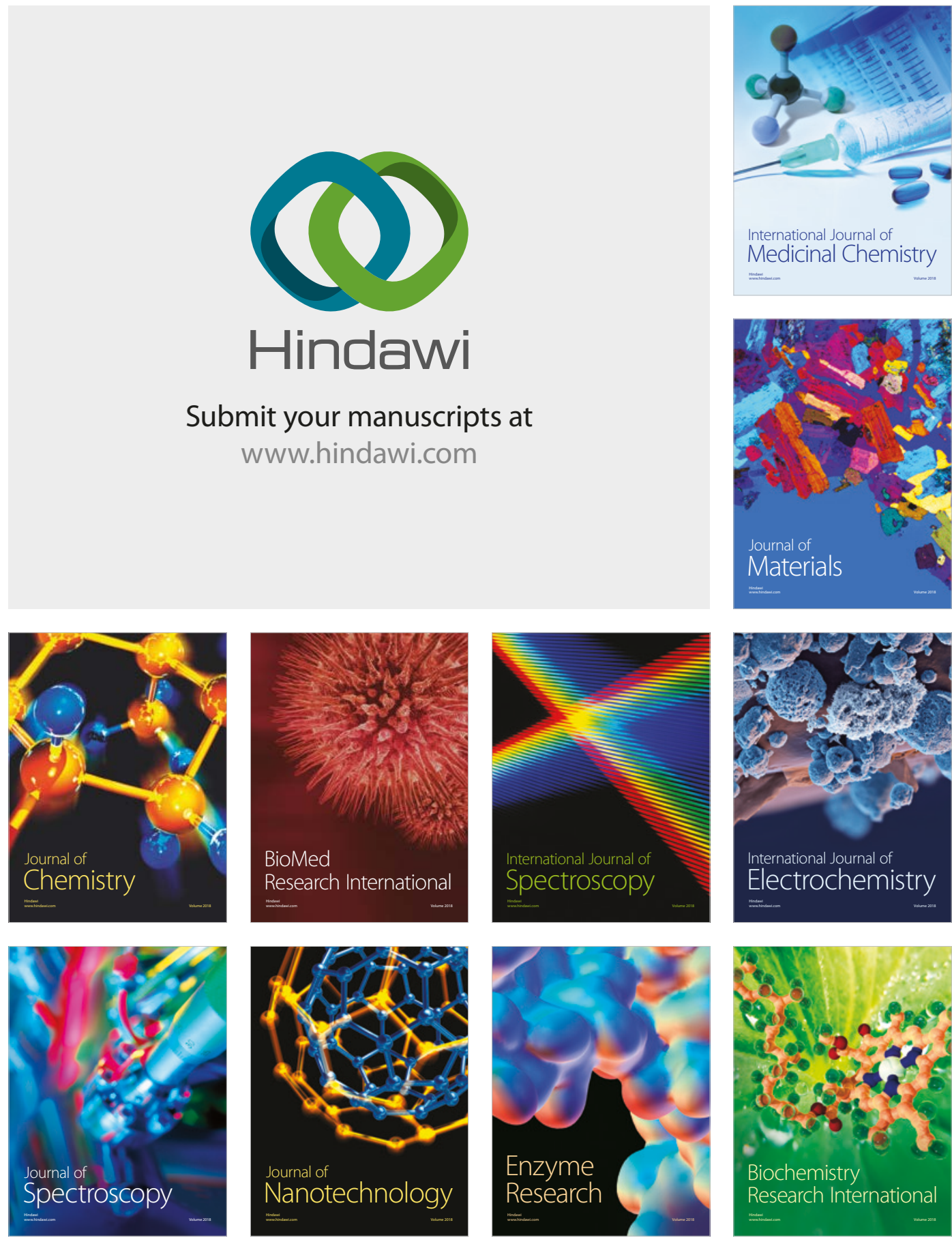
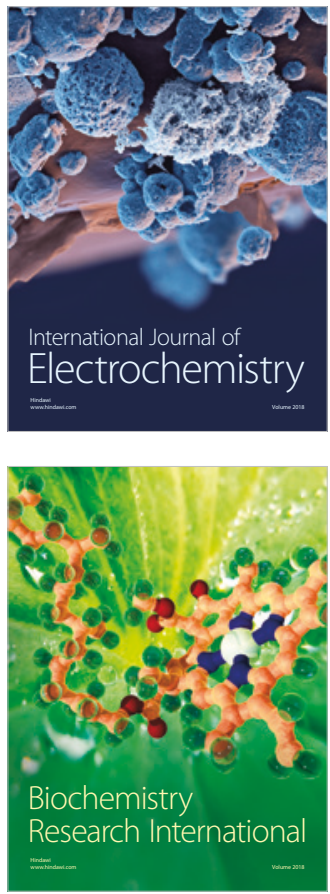